\title{
CONDITIONING MAPS ON ORTHOMODULAR LATTICES
}

\author{
by D. J. FOULIS $†$ AND C. H. RANDALL
}

(Received 12 September, 1969)

1. Introduction. Let $(X, \Sigma, \mu)$ be a probability space, so that $X$ is a non-empty set, $\Sigma$ is a Boolean $\sigma$-algebra of subsets of $X$, and $\mu$ is a probability measure defined on $\Sigma$. If $D \in \Sigma$ is such that $\mu(D) \neq 0$, then one traditionally associates with $D$ a new probability measure $\mu_{D}$, called the conditional probability measure determined by $D$, and defined by $\mu_{D}(E)=$ $\mu(D \cap E) / \mu(D)$, for all $E \in \Sigma$.

Define mappings $\gamma_{D}: \Sigma \rightarrow \Sigma$ and $\gamma_{D}^{+}: \Sigma \rightarrow \Sigma$ by $\gamma_{D}(E)=D \cap E$ and $\gamma_{D}^{+}(E)=D^{\prime} \cup E$, for all $E \in \Sigma$, where $D^{\prime}$ denotes the complement of $D$ in $X$. Then, we have $\gamma_{D}(E) \subset F \Leftrightarrow E \subset \gamma_{D}^{+}(F)$, for all $E, F \in \Sigma$. Moreover, if $E, F \in \Sigma$ with $E \subset F^{\prime}$, then $\gamma_{D}(E) \subset\left(\gamma_{D}(F)\right)^{\prime}$. Finally, $\mu_{D}(E)=$ $\mu\left(\gamma_{D}(E)\right) / \mu\left(\gamma_{D}(X)\right)$ holds for all $E \in \Sigma$.

In what follows, we shall generalize mappings such as $\gamma_{D}$ above from Boolean $\sigma$-algebras such as $\Sigma$ to arbitrary orthomodular lattices, our motivation being that the admissible propositions affiliated with an empirical science tend to band together to form an orthomodular lattice $L$, and such an $L$ need not be a Boolean algebra [6], [7], [8].

We shall assume that the reader is familiar with the basic facts about orthomodular lattices such as can be found in [1] and [4]. In particular, whenever we distribute an infimum over a supremum (or vice-versa) in the course of our calculations within an orthomodular lattice, it will be seen that this distribution is justified by [4, Theorem 5].

A map $\gamma: L_{0} \rightarrow L_{1}$, where $L_{0}$ and $L_{1}$ are orthomodular lattices, will be said to be residuated [3] if and only if there exists a second map $\gamma^{+}: L_{1} \rightarrow L_{0}$ (necessarily unique and called the residual of $\gamma$ ) such that, for all $e \in L_{0}$ and all $f \in L_{1}, \gamma(e) \leqq f \Leftrightarrow e \leqq \gamma^{+}(f)$. It is easy to see that a residuated map preserves arbitrary suprema and that the composition of residuated maps is again a residuated map; see [3]. If $\gamma: L_{0} \rightarrow L_{1}$ is residuated, we define the adjoint of $\gamma$ to be the map $\gamma^{*}: L_{1} \rightarrow L_{0}$ given by $\gamma^{*}(f)=\left(\gamma^{+}\left(f^{\prime}\right)\right)^{\prime}$, for all $f \in L_{1}$. Clearly, if $\gamma: L_{0} \rightarrow L_{1}$ is residuated and $e \in L_{1}$, then $\gamma(e)=0 \Leftrightarrow e \leqq\left(\gamma^{*}(1)\right)^{\prime}, 1$ being the order unit in $L_{1}$.

Two elements $e, f$ belonging to an orthomodular lattice are said to be orthogonal, in symbols $e \perp f$, if and only if $e \leqq f^{\prime}$. Two residuated maps $\gamma, \delta: L_{0} \rightarrow L_{1}$ are called orthogonal, in symbols $\gamma \perp \delta$, if and only if $\gamma(1)$ is orthogonal to $\delta(1)$. Evidently, $\gamma \perp \delta$ if and only if $\delta^{*} \gamma=0$, where $0: L_{0} \rightarrow L_{0}$ is the residuated map sending every element of $L_{0}$ onto the order zero $0 \in L_{0}$.

Suppose that $L_{0}, L_{1}$ are complete orthomodular lattices and that $\left(\gamma_{i} \mid i \in I\right)$ is a family of residuated maps $\gamma_{i}: L_{0} \rightarrow L_{1}$. Then, we define the envelope of the family $\left(\gamma_{i} \mid i \in I\right)$, in symbols env $\left(\gamma_{i} \mid i \in I\right)$, by env $\left(\gamma_{1} \mid i \in I\right)=\gamma$, where $\gamma: L_{0} \rightarrow L_{1}$ is the map given by $\gamma(e)=\bigvee\left(\gamma_{i}(e) \mid i \in I\right)$ for $e \in L_{0}$. It is easy to verify that $\operatorname{env}\left(\gamma_{i} \mid i \in I\right)$ is residuated and that $\left(\operatorname{env}\left(\gamma_{i} \mid i \in I\right)\right)^{*}=$ env $\left(\gamma_{i}^{*} \mid i \in I\right)$.

If $L$ is any orthomodular lattice and if $e \in L$, then the Sasaki projection $\phi_{e}: L \rightarrow L$ is defined by $\phi_{e}(f)=e \wedge\left(e^{\prime} \vee f\right)$, for all $f \in L$. It is known [4] that $\phi_{e}$ is a residuated map with $\phi_{e}=$

$\dagger$ The work of the first author on this paper was supported by National Science Foundation grant GP-9005. 
$\left(\phi_{e}\right)^{*}=\phi_{e} \phi_{e}$. If $e, f \in L$, we say that $e$ commutes with $f$ and we write $e C f$ if and only if $\phi_{e}(f)=$ $e \wedge f$. If $e C f$ for every $f \in L$, then we say that $e$ belongs to the center of $L$ and we denote the center of $L$ by $C(L)$. If $C(L)=\{0,1\}$, then we say that $L$ is irreducible. The basic facts about commutativity and the centre of an orthomodular lattice can be found in [4] and will not be repeated here.

If $L_{0}, L_{1}$ are orthomodular lattices and if $\phi: L_{0} \rightarrow L_{1}$ is a mapping that preserves finite infima, finite suprema and the orthocomplementation, then we call $\phi$ a homomorphism and we define the kernel of $\phi$ by $\operatorname{ker}(\phi)=\phi^{-1}(0)$. Of course, a bijective homomorphism is called an isomorphism.

Evidently, the kernel of a homomorphism is a lattice ideal in the domain of that homomorphism. If $L$ is any orthomodular lattice and if $J$ is a lattice ideal in $L$, then we call $J$ a $p$-ideal if and only if $\phi_{e}(J) \subset J$ holds for every $e \in L$. The kernel of a homomorphism is a $p$-ideal and, conversely, any $p$-ideal is a homomorphism kernel. Naturally, an orthomodular lattice $L$ is called simple if and only if every non-zero homomorphism defined on $L$ is an isomorphism onto its image. Consequently, $L$ is simple if and only if $\{0\}$ and $L$ itself are the only $p$-ideals in $L$. Clearly, any simple orthomodular lattice $L$ is irreducible, since if $e \neq 0,1$ is an element in the center of $L$, then $J=\{x \in L \mid x \leqq e\}$ is a non-trivial $p$-ideal in $L$.

If $L$ is any orthomodular lattice and if $e \in L$, then a subset of $L$ of the form $L[0, e]=$ $\{x \in L \mid x \leqq e\}$ is called a segment in $L$. If $f \rightarrow f^{\prime}$ denotes the orthocomplementation on $L$, then the map $x \rightarrow x^{*}=x^{\prime} \wedge e$ is an orthocomplementation for the segment $L[0, e]$ and, equipped with this orthocomplementation, $L[0, e]$ is itself an orthomodular lattice.

2. Conditioning maps. Let $L_{0}, L_{1}$ be orthomodular lattices. A map $\gamma: L_{0} \rightarrow L_{1}$ is called a conditioning map if and only if $\gamma$ is residuated and, for all $e, f \in L_{0}, e \perp f \Rightarrow \gamma(e) \perp \gamma(f)$. We note that if $(X, \Sigma, \mu)$ is a probability space and if $D \in \Sigma$, then the map $\gamma_{D}: \Sigma \rightarrow \Sigma$ defined for $E \in \Sigma$ by $\gamma_{D}(E)=D \cap E$ is a conditioning map.

LeMma 1. Let $L_{0}, L_{1}$ be orthomodular lattices and let $\gamma: L_{0} \rightarrow L_{1}$ be a residuated map. Then, the following conditions are mutually equivalent.

(i) for $e \in L_{0}, \gamma(e) \perp \gamma\left(e^{\prime}\right)$;

(ii) for $e \in L_{0}, \gamma\left(e^{\prime}\right)=\gamma(e)^{\prime} \wedge \gamma(1)$;

(iii) for $e \in L_{0}, \gamma(e)^{\prime}=\gamma\left(e^{\prime}\right) \vee \gamma(1)^{\prime}$;

(iv) $\gamma$ is a conditioning map.

Proof. Suppose that (i) holds. Since $\gamma$ is residuated, it is isotone, and so $\gamma\left(e^{\prime}\right) \leqq \gamma(1)$; hence, by (i), $\gamma\left(e^{\prime}\right) \leqq \gamma(e)^{\prime} \wedge \gamma(1)$. Put $g=\gamma(e)^{\prime} \wedge \gamma(1) \wedge \gamma\left(e^{\prime}\right)^{\prime}$, and note that (by orthomodularity) condition (ii) will follow immediately if we can show that $g=0$. Now

$$
g^{\prime}=\gamma(e) \vee \gamma(1)^{\prime} \vee \gamma\left(e^{\prime}\right)=\gamma\left(e \vee e^{\prime}\right) \vee \gamma(1)^{\prime}=\gamma(1) \vee \gamma(1)^{\prime}=1 ;
$$

hence $g=0$ and (ii) holds. Suppose that (ii) holds and replace $e$ by $e^{\prime}$ in (ii) to obtain $\gamma(e)=\gamma\left(e^{\prime}\right)^{\prime} \wedge \gamma(1)$. Taking the orthocomplement of both sides of the latter equation yields (iii). Assume that (iii) holds and that $a, b \in L_{0}$ with $a \perp b$. Then $\gamma(a) \leqq \gamma\left(b^{\prime}\right) \leqq \gamma\left(b^{\prime}\right) \vee \gamma(1)^{\prime}=\gamma(b)^{\prime}$; hence (iv) obtains. That (iv) implies (i) is clear, and the proof is complete. 
Corollary 2. Let $L_{0}, L_{1}$ be orthomodular lattices and let $\gamma: L_{0} \rightarrow L_{1}$ be a conditioning map. Put $L_{2}=L_{1}[0, \gamma(1)]$. Then $\gamma: L_{0} \rightarrow L_{2}$ is a homomorphism. Hence if $\left(e_{i} \mid i \in I\right)$ is any family of elements of $L_{0}$ indexed by the non-empty set $I$ and if $e=\wedge\left(e_{i} \mid i \in I\right)$ exists in $L_{0}$, then $\bigwedge\left(\gamma\left(e_{i}\right) \mid i \in I\right)$ exists in $L_{1}$ and equals $\gamma(e)$.

Lemma 3. Let $L_{0}, L_{1}$ be orthomodular lattices and let $\gamma: L_{0} \rightarrow L_{1}$ be a conditioning map. Then

(i) for $e \in L_{0}, \gamma^{*} \gamma(e) \leqq e$;

(ii) for $f \in L_{1}, f \wedge \gamma(1) \leqq \gamma \gamma^{*}(f)$;

(iii) for $e \in L_{0}, e \leqq \gamma^{*}(1) \Rightarrow e=\gamma^{*} \gamma(e)$;

(iv) if $\gamma^{*}(1)=1$, then $e=\gamma^{*} \gamma(e)=\gamma^{+} \gamma(e)$ for all $e \in L_{0}$.

Proof. To prove (i), we use part (iii) of Lemma 1 and compute as follows:

$$
\gamma^{*} \gamma(e)=\left(\gamma^{+}\left(\gamma(e)^{\prime}\right)\right)^{\prime}=\left(\gamma^{+}\left(\gamma\left(e^{\prime}\right) \vee \gamma(1)^{\prime}\right)\right)^{\prime} \leqq\left(\gamma^{+} \gamma\left(e^{\prime}\right)\right)^{\prime} \leqq e .
$$

To prove (ii), we make a similar computation, using part (ii) of Lemma 1, as follows:

$$
\gamma \gamma^{*}(f)=\gamma\left(\left(\gamma^{+}\left(f^{\prime}\right)\right)^{\prime}\right)=\left(\gamma \gamma^{+}\left(f^{\prime}\right)\right)^{\prime} \wedge \gamma(1) \geqq f \wedge \gamma(1) \text {. }
$$

To prove (iii), assume that $e \leqq \gamma^{*}(1)$ and put $g=\left(\gamma^{*} \gamma(e)\right)^{\prime} \wedge e$. By part(i) of the present lemma and the orthomodularity of $L_{0}$, it will suffice to show that $g=0$. We have

$$
\gamma(g)=\gamma\left(\left(\gamma^{*} \gamma(e)\right)^{\prime}\right) \wedge \gamma(e)=\left(\gamma \gamma^{*} \gamma(e)\right)^{\prime} \wedge \gamma(1) \wedge \gamma(e)
$$

by Corollary 2 and part (ii) of Lemma 1. By part (ii) of the present lemma, $\gamma(e) \wedge \gamma(1) \leqq \gamma \gamma^{*} \gamma(e)$; hence $\gamma(g)=0$. It follows that $g \leqq\left(\gamma^{*}(1)\right)^{\prime}$. Since also $g \leqq e \leqq \gamma^{*}(1)$, we have $g=0$ as desired.

To prove (iv), assume that $\gamma^{*}(1)=1, e \in L_{0}$. By part (iii) of the present lemma, we have $e=\gamma^{*} \gamma(e)$. Also, $e \leqq \gamma^{+} \gamma(e)$. Put $h=\gamma^{+} \gamma(e) \wedge e^{\prime}$, and note that (iv) will follow from the orthomodularity of $L_{0}$ if we can show that $h=0$. But,

$$
\gamma(h)=\gamma \gamma^{+} \gamma(e) \wedge \gamma\left(e^{\prime}\right)=\gamma(e) \wedge \gamma\left(e^{\prime}\right)=\gamma\left(e \wedge e^{\prime}\right)=\gamma(0)=0
$$

by Corollary 2. Hence, $h \leqq\left(\gamma^{*}(1)\right)^{\prime}=1^{\prime}=0$, and so $h=0$ as desired. The proof is complete.

LEMMA 4. Let $L_{0}, L_{1}$ be orthomodular lattices and let $\gamma: L_{0} \rightarrow L_{1}$ be a conditioning map. Then $\gamma^{*}(1)$ belongs to the center of $L_{0}$.

Proof. Let $e \in L_{0}$ and put $g=\gamma^{*}(1), h=\left(e \vee g^{\prime}\right) \wedge g$. We must show that $h=e \wedge g$. Since $e \wedge g \leqq h \leqq g$, it will suffice to prove that $h \leqq e$. By part (ii) of Lemma 3,

$$
\gamma(1)=1 \wedge \gamma(1) \leqq \gamma \gamma^{*}(1)=\gamma(g) \text {. }
$$

Since $\gamma(g) \leqq \gamma(1)$, we have $\gamma(g)=\gamma(1)$. Since $h \leqq g=\gamma^{*}(1)$, then, by part (iii) of Lemma $3, h=\gamma^{*} \gamma(h)$. But, since $\gamma(e) \leqq \gamma(1)=\gamma(g)$ and since $\gamma\left(g^{\prime}\right) \leqq \gamma(g)^{\prime}$, Corollary 2 gives $\gamma(h)=\left(\gamma(e) \vee \gamma\left(g^{\prime}\right)\right) \wedge \gamma(g)=\gamma(e) \wedge \gamma(g)=\gamma(e)$. It follows that $h=\gamma^{*} \gamma(h)=\gamma^{*} \gamma(e) \leqq e$ by part (i) of Lemma 3, and the proof is complete.

Corollary 5. Let $L_{0}, L_{1}$ be orthomodular lattices and let $L_{0}$ be irreducible. Let $\gamma: L_{0} \rightarrow L_{1}$ be a conditioning map other than the trivial map sending every element of $L_{0}$ onto the zero element of $L_{1}$. Then, for $e \in L_{0}, e=\gamma^{*} \gamma(e)=\gamma^{+} \gamma(e)$ and $\gamma$ is an injection.

Proof. Lemma 4 and part (iv) of Lemma 3. 
LEMMA 6. Let $L_{0}, L_{1}$ be orthomodular lattices and let $\gamma: L_{0} \rightarrow L_{1}$ be a conditioning map. Then, for $e, f \in L_{0}, \gamma\left(\phi_{e}(f)\right)=\phi_{\gamma(e)}(\gamma(f))$. we have

Proof. Since $\gamma(e), \gamma\left(e^{\prime}\right), \gamma(f) \leqq \gamma(1)$, and since $\gamma(e)^{\prime}=\gamma\left(e^{\prime}\right) \vee \gamma(1)^{\prime}$ by part (iii) of Lemma 1,

$$
\begin{aligned}
\phi_{\gamma(e)}(\gamma(f)) & =\gamma(e) \wedge\left(\gamma(e)^{\prime} \vee \gamma(f)\right) \\
& =\gamma(e) \wedge\left(\gamma\left(e^{\prime}\right) \vee \gamma(f) \vee \gamma(1)^{\prime}\right) \\
& =\left[\gamma(e) \wedge\left(\gamma\left(e^{\prime}\right) \vee \gamma(f)\right)\right] \vee\left[\gamma(e) \wedge \gamma(1)^{\prime}\right] \\
& =\gamma\left(e \wedge\left(e^{\prime} \vee f\right)\right) \vee 0=\gamma\left(\phi_{e}(f)\right) .
\end{aligned}
$$

Corollary 7. Let $L_{0}, L_{1}$ be orthomodular lattices and let $\gamma: L_{0} \rightarrow L_{1}$ be a conditioning map. Let $e, f \in L_{0}$. Then

(i) $e C f \Rightarrow \gamma(e) C \gamma(f)$;

(ii) if $\gamma^{*}(1)=1$, then $e C f \Leftrightarrow \gamma(e) C \gamma(f)$.

Lemma 8. Let $L_{0}, L_{1}$ be complete orthomodular lattices and let $\left(\gamma_{i} \mid i \in I\right)$ be an orthogonal family of conditioning maps $\gamma_{i}: L_{0} \rightarrow L_{1}$. Then env $\left(\gamma_{i} \mid i \in I\right)=\gamma$ is a conditioning map.

Proof. Let $e \in L_{0}$. By part (i) of Lemma 1, it will suffice to prove that $\gamma\left(e^{\prime}\right) \leqq \gamma(e)^{\prime}$; that is,

$$
\bigvee\left(\gamma_{i}\left(e^{\prime}\right) \mid i \in I\right) \leqq \bigwedge\left(\gamma_{j}(e)^{\prime} \mid j \in I\right)
$$

To prove the latter inequality, we must show that, for $i, j \in I, \gamma_{i}\left(e^{\prime}\right) \leqq \gamma_{j}(e)^{\prime}$. If $i=j$, this is clear from the fact that $\gamma_{i}$ is a conditioning map; hence we can suppose that $i \neq j$. Then, since $\left(\gamma_{i} \mid i \in I\right)$ is an orthogonal family, $\gamma_{i}(1) \leqq \gamma_{j}(1)^{\prime}$; hence $\gamma_{i}(e) \leqq \gamma_{i}(1) \leqq \gamma_{j}(1)^{\prime} \leqq \gamma_{j}(e)^{\prime}$. The proof is complete.

LEMma 9. Let $L_{0}, L_{1}$ be orthomodular lattices and let $\gamma: L_{0} \rightarrow L_{1}$ be a conditioning map. Then, if $J$ is a p-ideal in $L_{1}, \gamma^{-1}(J)$ is a p-ideal in $L_{0}$.

Proof. Since $J$ is a $p$-ideal in $L_{1}$, we can form the quotient orthomodular lattice $L_{1} / J$. Let $\eta$ be the canonical homomorphism $\eta: L_{1} \rightarrow L_{1} / J$ and define a map

$$
\phi: L_{1}[0, \gamma(1)] \rightarrow L_{1} / J[0, \eta \gamma(1)]
$$

by $\phi(f)=\eta(f)$ for all $f \in L_{1}[0, \gamma(1)]$. Evidently, $\phi$ is a homomorphism and $\operatorname{ker}(\phi)=$ $J \cap L_{1}[0, \gamma(1)]$. Hence $\phi \gamma: L_{0} \rightarrow L_{1} / J[0, \eta \gamma(1)]$ is a homomorphism, so $\gamma^{-1}(J)=\operatorname{ker}(\phi \gamma)$ is a p-ideal.

3. Complete Dacey spaces. By an orthogonality space, we mean an ordered pair $(X, \perp)$ where $X$ is a non-empty set and $\perp$ is a symmetric irreflexive binary relation defined on $X$. If $(X, \perp)$ is an orthogonality space and $A \subset X$, we define $A^{\perp}=\{x \in X \mid x \perp a$ for all $a \in A\}, A^{\perp \perp}=$ $\left(A^{\perp}\right)^{\perp}$, etc. For $A, B \subset X$, we always have $A \subset A^{\perp \perp}$ and $A \subset B \Rightarrow B^{\perp} \subset A^{\perp}$; hence $A^{\perp}=A^{\perp \perp \perp}$. A subset $C$ of $X$ is called closed if and only if $C=C^{\perp \perp}$ and the set of all closed subsets of $X$ is denoted by $\mathscr{C}(X, \perp)$. Evidently, $\emptyset, X \in \mathscr{C}(X, \perp)$ and, for $A \subset X, A \in \mathscr{C}(X, \perp)$ if and only if there exists $B \subset X$ such that $B^{\perp}=A$. Partially ordered by ordinary set inclusion and equipped with the orthocomplementation $C \rightarrow C^{\perp}, \mathscr{C}(X, 1)$ forms a complete ortholattice [1]. If $\left(C_{j}\right)$ is any 
family of elements of $\mathscr{C}(X, \perp)$, then the infimum and the supremum of the family $\left(C_{j}\right)$ are given respectively by the formulas

$$
\bigwedge_{j} C_{j}=\bigcap_{j} C_{j} \text { and } \bigvee_{j} C_{j}=\left(\bigcup_{j} C_{j}\right)^{\perp \perp}=\left(\bigcap_{j} C_{j}^{\perp}\right)^{\perp}
$$

A subset $D$ of $X$ is called an orthogonal set if and only if $a, b \in D \Rightarrow a=b$ or $a \perp b$. If $A \subset B \subset X$ and if $A$ is an orthogonal set, then (by Zorn's lemma) there is a maximal orthogonal set $D \subset B$ such that $A \subset D$.

We call $(X, \perp)$ a complete Dacey space [2] if and only if whenever $A \in \mathscr{C}(X, \perp)$ and $D$ is a maximal orthogonal subset of $A$, then $D^{\perp \perp}=A^{\perp \perp}$. By [5, Theorem 1], $(X, \perp)$ is a complete Dacey space if and only if $\mathscr{C}(X, \perp)$ is a complete orthomodular lattice.

Let $(X, \#)$ be any orthogonality space and let $\Gamma$ denote the free monoid (semigroup with unit 1) over $X$. We extend the orthogonality relation $\#$ on $X$ to an orthogonality relation $\perp$ on $\Gamma$ by defining $a \perp b$ (for $a, b \in \Gamma$ ) if and only if there exist $c, d, e \in \Gamma$ and there exist $x, y \in X$ with $a=c x d, b=c y e$ and $x \# y$. In [5, Theorem 4], we proved that if $(X, \#)$ is a complete Dacey space, then so is $(\Gamma, \perp)$. We call $(\Gamma, \perp)$ the free orthogonality monoid over the base space $(X, \#)$. The motivation for this construction can be found in [8] and will not be repeated here.

Henceforth we assume, once and for all, that $(X, \#)$ is a complete Dacey space and that $(\Gamma, \perp)$ is the free orthogonality monoid over $(X, \#)$. Motivated by [8], we refer to an orthogonal subset $D$ of $\Gamma$ as an event and we call a maximal event $E$ an operation. If $A, B \subset \Gamma$, we naturally define $A B=\{a b \mid a \in A$ and $b \in B\}$ and we note that the product of two events is again an event. We do not bother to distinguish between a singleton subset $\{a\}$ of $\Gamma$ and the element $a \in \Gamma$, so that, for instance, we write $\{a\} B$ as $a B$. For $a \in \Gamma, B \subset \Gamma$, we define $a^{-1} B \subset \Gamma$ by $a^{-1} B=\{c \in \Gamma \mid a c \in B\}$, and we note that if $D$ is an event, so is $a^{-1} D$. Furthermore, if $D$ is an event and $a^{-1} D \neq \emptyset$, one easily verifies that $\left(a^{-1} D\right)^{\perp}=a^{-1} D^{\perp}$; hence, if $E$ is an operation and $a^{-1} E \neq \emptyset$, then $a^{-1} E$ is again an operation. The following lemma can be proved by direct calculation.

Lemma 10. Let $D$ be a non-empty event and suppose that, for each $d \in D, M_{d}$ is a non-empty subset of $\Gamma$. Let $D_{0}=\left\{d \in D \mid M_{d}^{\perp} \neq \emptyset\right\}$. Put $M=\bigcup\left(d M_{d} \mid d \in D\right)$. Then

(i) $M^{\perp}=\bigcup\left(d M_{d}^{\perp} \mid d \in D_{0}\right) \cup D^{\perp}$,

(ii) $M^{\perp \perp}=\bigcup\left(d M_{d}^{\perp \perp} \mid d \in D_{0}\right) \cup\left(D \backslash D_{0}\right)^{\perp \perp}$.

CoRollary 11. Let $D$ be any event and let $B \subset \Gamma$. Then

(i) if $B \neq \emptyset,(D B)^{\perp}=D B^{\perp} \cup D^{\perp}$;

(ii) if $B^{\perp} \neq \emptyset,(D B)^{\perp \perp}=D B^{\perp \perp}$;

(iii) if $B^{\perp}=\emptyset,(D B)^{\perp \perp}=D^{\perp \perp}$.

We now define a mapping $\Psi: \mathscr{C}(X, \#) \rightarrow \mathscr{C}(\Gamma, \perp)$ by $\Psi(A)=A^{\perp \perp}$ for $A=A^{\# \sharp} \in \mathscr{C}(X, \sharp)$. It is easy to verify that $\Psi$ is a conditioning map and that its adjoint is given by $\Psi^{*}(B)=$ 
$\left(B^{\perp} \cap X\right)^{*}$ for all $B=B^{\perp \perp} \in \mathscr{C}(\Gamma, \perp)$. Furthermore, $\Psi(X)=\Gamma$ and $\Psi^{*}(\Gamma)=X$; hence $\Psi: \mathscr{C}(X, \#) \rightarrow \mathscr{C}(\Gamma, \perp)$ is not only a conditioning map, but also an injective homomorphism. Notice that if $Z \subset X, \Psi\left(Z^{\# *}\right)=Z^{\perp \perp}$. We shall refer to the map $\Psi$ as the canonical embedding of $\mathscr{C}(X, \#)$ into $\mathscr{C}(\Gamma, \perp)$.

We omit the straightforward proof of the following lemma.

LEMmA 12. Let $\Psi: \mathscr{C}(X, \#) \rightarrow \mathscr{C}(\Gamma, \perp)$ be the canonical embedding. Let $Z \subset X$. Then

(i) $\Psi\left(Z^{* *}\right)=Z^{\perp \perp}$;

(ii) $\Psi\left(Z^{*}\right)=Z^{\perp}$;

(iii) if $Z^{\sharp} \neq \emptyset, \Psi\left(Z^{\# *}\right)=Z^{\sharp \#} \Gamma$.

For $d \in \Gamma$, we define a mapping $\gamma_{d}: \mathscr{C}(\Gamma, \perp) \rightarrow \mathscr{C}(\Gamma, \perp)$ by $\gamma_{d}(A)=(d A)^{\perp \perp}$ for $A=$ $A^{\perp \perp} \in \mathscr{C}(\Gamma, \perp)$. By Corollary 11 , we have

$$
\gamma_{d}(A)=\left\{\begin{array}{lll}
d A & \text { if } & A \neq \Gamma, \\
d^{\perp \perp} & \text { if } & A=\Gamma,
\end{array}\right.
$$

for all $d \in \Gamma$ and all $A \in \mathscr{C}(\Gamma, \perp)$.

Lemma 13. If $A \in \mathscr{C}(\Gamma, \perp)$ and if $b \in \Gamma$, then $b^{-1} A \in \mathscr{C}(\Gamma, \perp)$.

Proof. Let $e \in \Gamma$. If $e \in b^{\perp}$, we have $b^{-1} e^{\perp}=\Gamma$. If $e \in b \Gamma$, say $e=b d$ for some $d \in \Gamma$, then $b^{-1} e^{\perp}=d^{\perp}$. If $e \notin b \Gamma \cup b^{\perp}$, then $b^{-1} e^{\perp}=\emptyset$. In any case, $b^{-1} e^{\perp} \in \mathscr{C}(\Gamma, \perp)$. Since $A$ is closed, we have $A=\bigcap\left(e^{\perp} \mid e \in A^{\perp}\right)$; hence $b^{-1} A=\bigcap\left(b^{-1} e^{\perp} \mid e \in A^{\perp}\right)$. Since an intersection of closed sets is closed, the lemma is proved.

THEOREM 14. For each $d \in \Gamma$, the map $\gamma_{d}: \mathscr{C}(\Gamma, \perp) \rightarrow \mathscr{C}(\Gamma, \perp)$ is a conditioning map and its residual $\gamma_{d}^{+}$is given by $\gamma_{d}^{+}(A)=d^{-1} A$.

Proof. By Lemma 13, the map $\gamma_{d}^{+}: \mathscr{C}(\Gamma, \perp) \rightarrow \mathscr{C}(\Gamma, \perp)$ is well-defined. Evidently, for $A, B \in \mathscr{C}(\Gamma, \perp)$, we have $\gamma_{d}(A) \leqq B \Leftrightarrow A \leqq \gamma_{d}^{+}(B)$; hence $\gamma_{d}$ is residuated with $\gamma_{d}^{+}$as its residual. For $A \in \mathscr{C}(\Gamma, \perp)$, we have $d A^{\perp} \subset(d A)^{\perp}$, so that $\left(d A^{\perp}\right)^{\perp \perp} \subset(d A)^{\perp \perp \perp}$; that is, $\gamma_{d}\left(A^{\perp}\right) \subset \gamma_{d}(A)^{\perp}$. It follows from part (i) of Lemma 1 that $\gamma_{d}$ is a conditioning map.

For $d \in \Gamma$, we have $\gamma_{d}(\Gamma)=d^{\perp \perp}$; hence two conditioning maps $\gamma_{d}$ and $\gamma_{e}$ are orthogonal if and only if $d \perp e$ in $\Gamma$. Consequently, if $D$ is an event, then the family $\left(\gamma_{d} \mid d \in D\right)$ is an orthogonal family of conditioning maps, so by Lemma 8, env $\left(\gamma_{d} \mid d \in D\right)$ is again a conditioning map. For any event $D$ we define $; o: \mathscr{C}(\Gamma, \perp) \rightarrow \mathscr{C}(\Gamma, \perp)$ by $\gamma_{D}=\operatorname{env}\left(\gamma_{d} \mid d \in D\right)$. Evidently, for any event $D$ and any $A \in \mathscr{C}(\Gamma, \perp), \gamma_{D}(A)=(D A)^{\perp \perp}$ and $\gamma_{D}(\Gamma)=D^{\perp \perp}$.

Lemma 15. Let $D$ be an event. Then, $\gamma_{D}^{*}(\Gamma)=\Gamma$. Hence $\gamma_{D}: \mathscr{C}(\Gamma, \perp) \rightarrow \mathscr{C}(\Gamma, \perp)$ is an injection preserving arbitrary infima and suprema as well as orthogonality. Also, if $A, B \in \mathscr{C}(\Gamma, \perp)$, then $A$ commutes with $B$ in $\mathscr{C}(\Gamma, \perp)$ if and only if $\gamma_{D}(A)$ commutes with $\gamma_{D}(B)$ in $\mathscr{C}(\Gamma, 1)$.

Proof. For any $A \in \mathscr{C}(\Gamma, \perp)$, we have $\gamma_{D}^{*}(A)=\bigvee\left(\left(d^{-1} A^{\perp}\right)^{\perp} \mid d \in D\right)$; hence $\gamma_{D}^{*}(\Gamma)=\Gamma$. Application of part (iv) of Lemma 3 and part (ii) of Corollary 7 completes the proof. 
Lemma 16. Let $d \in \Gamma, A \in \mathscr{C}(\Gamma, \perp)$ and suppose that $\gamma_{d}^{+}(A) \neq \emptyset$. Then $\gamma_{d}^{+}\left(A^{\perp}\right)=\left(\gamma_{d}^{+}(A)\right)^{\perp}$.

Proof. Suppose that $e \in d^{-1} A$, so that $d e \in A$. We must show that $d^{-1} A^{\perp}=\left(d^{-1} A\right)^{\perp}$. Since it is clear that $d^{-1} A^{\perp} \subset\left(d^{-1} A\right)^{\perp}$, it will suffice to show that $\left(d^{-1} A\right)^{\perp} \subset d^{-1} A^{\perp}$. Let $D$ be a maximal orthogonal subset of $A$ chosen so that $\operatorname{de} \in D$. Then $D^{\perp \perp}=A, D^{\perp}=A^{\perp}$ and $e \in d^{-1} D$, so that $\emptyset \neq d^{-1} D$. Since $\emptyset \neq d^{-1} D$, then $\left(d^{-1} D\right)^{\perp}=d^{-1} D^{\perp}=d^{-1} A^{\perp}$; hence it will suffice to show that $\left(d^{-1} A\right)^{\perp} \subset\left(d^{-1} D\right)^{\perp}$. Since $D \subset A$, then $d^{-1} D \subset d^{-1} A$ and $\left(d^{-1} A\right)^{\perp} \subset$ $\left(d^{-1} D\right)^{\perp}$ as required.

Corollary 17. Suppose that $A, B \in \mathscr{C}(\Gamma, \perp)$ and that $A$ commutes with $B$ in $\mathscr{C}(\Gamma, \perp)$. Then, for every $d \in \Gamma, \gamma_{d}^{+}(A)$ commutes with $\gamma_{d}^{+}(B)$ in $\mathscr{C}(\Gamma, \perp)$.

Proof. We can assume that $\gamma_{d}^{+}(A) \neq \emptyset$. Hence, by Lemma 16, $\left(\gamma_{d}^{+}(A)\right)^{\perp}=\gamma_{d}^{+}\left(A^{\perp}\right)$. Since $\gamma_{d}^{+}$is an isotone map, $\gamma_{d}^{+}\left(A^{\perp}\right) \vee \gamma_{d}^{+}(B) \leqq \gamma_{d}^{+}\left(A^{\perp} \vee B\right)$. Thus

$$
\begin{aligned}
\gamma_{d}^{+}(A) \wedge \gamma_{d}^{+}(B) & \leqq \gamma_{d}^{+}(A) \wedge\left[\left(\gamma_{d}^{+}(A)\right)^{\perp} \vee \gamma_{d}^{+}(B)\right] \\
& =\gamma_{d}^{+}(A) \wedge\left[\gamma_{d}^{+}\left(A^{\perp}\right) \vee \gamma_{d}^{+}(B)\right] \\
& \leqq \gamma_{d}^{+}(A) \wedge \gamma_{d}^{+}\left(A^{\perp} \vee B\right) \\
& =\gamma_{d}^{+}\left(A \wedge\left(A^{\perp} \vee B\right)\right) \\
& =\gamma_{d}^{+}(A \wedge B)=\gamma_{d}^{+}(A) \wedge \gamma_{d}^{+}(B)
\end{aligned}
$$

It follows that $\gamma_{d}^{+}(A)$ commutes with $\gamma_{d}^{+}(B)$.

THEOREM 18. If $\mathscr{C}(X, \sharp)$ is a simple orthomodular lattice, then so is $\mathscr{C}(\Gamma, \perp)$.

Proof. Suppose that $\mathscr{C}(X, \sharp)$ is simple but that $\mathscr{C}(\Gamma, \perp)$ is not. Then $\mathscr{C}(\Gamma, \perp)$ contains a non-trivial $p$-ideal, $\mathscr{I}$ say. Then there exists $A \in \mathscr{C}(\Gamma, \perp)$ with $A \neq \emptyset, A \neq \Gamma$ and $A \in \mathscr{I}$. Since $A \neq \emptyset$, we can choose an element $a \in A$. Since $A \neq \Gamma$, then $a^{\perp} \neq \emptyset$. Since $a^{\perp \perp} \subset A^{\perp \perp}=$ $A \in \mathscr{I}$, then $a^{\perp \perp} \in \mathscr{I}$.

Every element $a \in \Gamma$, other than the unit 1, can be written uniquely in the form $a=$ $x_{1} x_{2} \ldots x_{n}$ with $x_{1}, x_{2}, \ldots, x_{n} \in \Gamma$. We define length $(a)=n$ and we define length $(1)=0$. For each non-trivial $p$-ideal $\mathscr{I}$ in $\mathscr{C}(\Gamma, \perp)$, we define $n(\mathscr{I})=\min \left(\right.$ length $(a) \mid a \in \Gamma, a^{\perp} \neq \emptyset$ and $\left.a^{\perp \perp} \in \mathscr{I}\right)$. Choose $\mathscr{I}_{0}$ to be a non-trivial $p$-ideal in $\mathscr{C}(\Gamma, \perp)$ for which $n\left(\mathscr{I}_{0}\right)=n_{0}$ is minimal and choose $a \in \Gamma$ with $a^{\perp} \neq \emptyset, a^{\perp \perp} \in \mathscr{I}_{0}$ and length $(a)=n_{0}$. Since $a^{\perp} \neq \emptyset$, then $a \neq 1$; hence we can factor $a$ as $a=x b$ for some $x \in X$ and some $b \in \Gamma$.

Let $\Psi: \mathscr{C}(X, \sharp) \rightarrow \mathscr{C}(\Gamma, \perp)$ be the canonical embedding and put $\mathscr{J}_{0}=\Psi^{-1}\left(\mathscr{I}_{0}\right)$. By Lemma $9, \mathscr{J}_{0}$ is a $p$-ideal in $\mathscr{C}(X, \#)$; hence (since $\mathscr{C}(X, \#)$ is simple) $\mathscr{J}_{0}=\{\emptyset\}$ or else $\mathscr{J}_{0}=$ $\mathscr{C}(X, \sharp)$. In the latter case, we would have $\Gamma=\Psi(X) \in \mathscr{I}_{0}$, contradicting the non-triviality of $\mathscr{I}_{0}$; hence we conclude that $\mathscr{J}_{0}=\{\emptyset\}$.

If $b^{\perp}=\emptyset$, we would have $a^{\perp \perp}=x^{\perp \perp}$ so that $n_{0}=1$ and $a=x$. But then $x^{\# \#} \in \Psi^{-1}\left(\mathscr{I}_{0}\right)=$

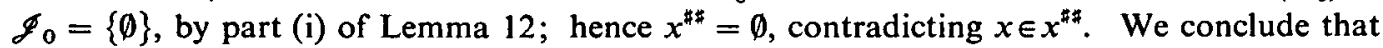
$b^{\perp} \neq \emptyset$. Hence $n_{0}=$ length $(a)=1+$ length $(b)>1$.

Since $b^{\perp} \neq \emptyset$, we have $\gamma_{x}\left(b^{\perp \perp}\right)=a^{\perp \perp} \in \mathscr{I}_{0}$. Let $\mathscr{I}_{1}=\gamma_{x}^{-1}\left(\mathscr{I}_{0}\right)$, noting that (by Lemma 9) $\mathscr{I}_{1}$ is a $p$-ideal in $\mathscr{C}(\Gamma, \perp)$ and that $b^{\perp \perp} \in \mathscr{I}_{1}$. If $\mathscr{I}_{1}=\mathscr{C}(\Gamma, \perp)$, then $x^{\perp \perp}=\gamma_{x}(\Gamma) \in \mathscr{I}_{0}$, con- 
tradicting $n\left(\mathscr{I}_{0}\right)=n_{0}>1$. Hence $\mathscr{I}_{1}$ is a non-trivial $p$-ideal in $\mathscr{C}(\Gamma, \perp)$ and $n\left(\mathscr{I}_{1}\right) \leqq$ length $(b)=n_{0}-1$, contradicting our choice of $\mathscr{I}_{0}$ and completing the proof.

If $C$ and $D$ are events, it is easy to check (using Corollary 11 ) that $\gamma_{C} \gamma_{D}=\gamma_{C D}$; hence the set of all $\gamma_{D}$ such that $D$ is an event forms a monoid under composition. This monoid is analogous to the Baer *-semigroup $S_{\Omega}$ obtained by Pool [6] in his axiomatization of general quantum mechanics; however, we shall not discuss the exact connection between this monoid of conditioning maps and Pool's $S_{\Omega}$ in this paper.

\section{REFERENCES}

1. G. Birkhoff, Lattice Theory, Amer. Math. Soc. Colloquium Pub. XXV (Providence, R.I., 1967).

2. J. C. Dacey, Jr., Orthomodular Spaces, Ph.D. Thesis, Univ. of Mass., Amherst, Mass., 1968.

3. J. C. Derdérian, Residuated mappings, Pacific J. Math. 20 (1967), 35-43.

4. D. J. Foulis, A note on orthomodular lattices, Portugaliae Math. 21, Fasc. 1 (1962), 65-72.

5. D. J. Foulis and C. H. Randall, Lexicographic orthogonality (to appear in J. Combinatorial Theory).

6. J. C. T. Pool, Baer *-semigroups and the logic of quantum mechanics, Commun. Math. Phys. 9 (1968), 118-141.

7. C. H. Randall, A Mathematical Foundation for Empirical Science with Special Reference to Quantum Theory, Knolls Atomic Power Lab. Report KAPL-3147 (1966).

8. C. H. Randall and D. J. Foulis, An approach to empirical logic, Amer. Math. Monthly 77 (1970), 363-374.

The UNIVERSITY OF MASSACHUSETTS

AMHERST, MASSACHUSETTS 